\title{
High Frequency Ultrasound for In Vivo Pregnancy Diagnosis and Staging of Placental and Fetal Development in Mice
}

\author{
Adelaide Greco ${ }^{1,2 *}$, Monica Ragucci ${ }^{2}$, Anna Rita Daniela Coda ${ }^{3}$, Alessandro Rosa ${ }^{1,2,3,4}$, Sara Gargiulo ${ }^{3}$, \\ Raffaele Liuzzi ${ }^{3}$, Matteo Gramanzini ${ }^{3}$, Sandra Albanese ${ }^{1,2,3,4}$, Sabina Pappatà ${ }^{3}$, Marcello Mancini ${ }^{3}$, \\ Arturo Brunetti ${ }^{1,2,3,49}$, Marco Salvatore ${ }^{19}$
}

1 Dipartimento di Scienze Biomediche Avanzate, Università degli studi di Napoli Federico II, Napoli, Italy, 2 IRCCS Fondazione SDN, Napoli, Italy, 3 Istituto di Biostrutture e Bioimmagini, CNR, Napoli, Italy, 4 Ceinge, Biotecnologie Avanzate, scarl, Napoli, Italy

\begin{abstract}
Background: Ultrasound is a valuable non-invasive tool used in obstetrics and gynecology to monitor the growth and well being of the human fetus. The laboratory mouse has recently emerged as an appropriate model for fetal and perinatal studies because morphogenetic processes in mice exhibit adequate homology to those in humans, and genetic manipulations are relatively simple to perform in mice. High-frequency ultrasound (HFUS) has recently become available for small animal preclinical imaging and can be used to study pregnancy and development in the mouse. The objective of the current study was to assess the main applications of HFUS in the evaluation of fetal growth and placental function and to better understand human congenital diseases.

Methodology/Principal Findings: On each gestational day, at least 5 dams were monitored with HFUS; a total of $\sim 200$ embryos were examined. Because it is not possible to measure each variable for the entire duration of the pregnancy, the parameters were divided into three groups as a function of the time at which they were measured. Univariate analysis of the relationship between each measurement and the embryonic day was performed using Spearman's rank correlation (Rs). Continuous linear regression was adopted for multivariate analysis of significant parameters. All statistical tests were twosided, and a $\mathrm{p}$ value of 0.05 was considered statistically significant.

Conclusions/Significance: The study describes the main applications of HFUS to assess changes in phenotypic parameters in the developing CD1 mouse embryo and fetus during pregnancy and to evaluating physiological fetal and placental growth and the development of principal organs such as the heart, kidney, liver, brain and eyes in the embryonic mouse. A database of normal structural and functional parameters of mouse development will provide a useful tool for the better understanding of morphogenetic and cardiovascular anomalies in transgenic and mutant mouse models.
\end{abstract}

Citation: Greco A, Ragucci M, Coda ARD, Rosa A, Gargiulo S, et al. (2013) High Frequency Ultrasound for In Vivo Pregnancy Diagnosis and Staging of Placental and Fetal Development in Mice. PLoS ONE 8(10): e77205. doi:10.1371/journal.pone.0077205

Editor: Ana Claudia Zenclussen, Medical Faculty, Otto-von-Guericke University Magdeburg, Medical Faculty, Germany

Received July 22, 2013; Accepted September 9, 2013; Published October 14, 2013

Copyright: (c) 2013 Greco et al. This is an open-access article distributed under the terms of the Creative Commons Attribution License, which permits unrestricted use, distribution, and reproduction in any medium, provided the original author and source are credited.

Funding: The authors have no support or funding to report.

Competing Interests: The authors have declared that no competing interests exist.

*E-mail: adegreco@unina.it

9 These authors contributed equally to this work.

\section{Introduction}

Real-time ultrasound imaging is routinely used for assessing pregnancy, fetal development and well being, as well as the effects of treatments that could alter these parameters in humans $[1,2]$. Common measurements performed by ultrasound to evaluate human fetal growth are outer-outer biparietal diameter (BPD), head circumference $(\mathrm{HC})$, mean abdominal diameter $(\mathrm{AD})$, abdominal circumference (AC) and femur length (FL) [1]. The laboratory mouse has emerged as an appropriate model for fetal and perinatal studies because morphogenetic processes in mice show adequate homology to those in humans. Moreover, mice are inexpensive, are easy to handle and house, and are often used for genetic manipulations. CD1 is the outbred laboratory mouse strain that is most widely used in basic reproductive biology studies in which genetic variability in the experimental population is desirable. In developmental studies, it is highly desirable to acquire longitudinal data in a non-invasive manner and with high spatial and temporal resolution and to define structural parameters [1-2]. Several imaging approaches have become available for the study of mouse development, including ultrasound, magnetic resonance, optical imaging and confocal biomicroscopy [3]. Advances in ultrasound technology have recently led to the development of high-frequency ultrasound (HFUS), which can be used for the high-resolution imaging of mouse embryonic development [4-10]. HFUS is a non-invasive, relatively safe and inexpensive molecular imaging technology.

Since the first study of mouse embryos in utero was performed by Turnbull et al. in 1995 [11], ultrasound has been used for both 
transcutaneous and transuterine imaging. Some studies employed conventional 13-15 MHz ultrasound systems [12], with spatial resolutions ranging from 200 to $500 \mu \mathrm{m}$. High-frequency systems (40-100 MHz), which offer a spatial resolution of approximately $30 \mu \mathrm{m}$ and penetration depths of several millimeters, are more often used for mouse embryo imaging. These sound waves are generally harmless at reasonable doses, particularly in the soft tissue environment of the embryo. Many studies have also reported the use of Doppler ultrasound for measuring the direction and velocity of blood flow. The change in the frequency of sound waves as they reflect off moving blood cells produces a Doppler effect, and the shift in frequency between the transmitted and received waves can be used to calculate the velocity of the moving blood $[3,13]$. HFUS appears to be a practical tool that can be used to determine gestational age, to monitor placental and fetal growth and embryonic or fetal viability, to evaluate morphological development, and to assess physiological cardiovascular structure in the mouse embryo and fetus throughout pregnancy. Its use would also contribute to improved animal welfare, in accordance with the philosophy of the 3 Rs model of Russell and Burch (Refinement, Reduction and Replacement). Early confirmation of pregnancy or fetal wastage in valuable animals can be used as evidence for delayed development or growth retardation in mutant or manipulated animals compared with wild-type animals [5]. Transcutaneous images of implantation sites can be obtained in pregnant mice at 5 days of gestation. At embryonic day (E) 6.5, implantation sites are clearly visible as regions in which the crosssection of the uterus is enlarged, and a small, echo-free region, which is likely the proamniotic cavity of the developing embryo, is detectable within the center of each enlargement. By E7.5, three dark regions are visible within the conceptus, corresponding to the ectoplacental, amniotic and exocoelomic cavities of the developing embryo. At E 8.5, the embryo and amniotic membrane are visible, and the allantois can be observed emerging from the embryo and approaching the ectoplacental cone, where the chorioallantoic placenta later develops. On day 9.5, the amniotic membrane, amniotic and yolk sac cavities, brain, cerebral ventricles and heart are visible. HFUS allows the accurate characterization of morphological details specific to each stage of development. Over the past several years, a number of authors have demonstrated the effective use of ultrasound backscatter microscopy to evaluate the development of the central nervous system, eye, and heart in fetal mice [14-18]. Later during embryonic development, many other structures can also be visualized using transcutaneous imaging, including the developing paw and forelimb, eyes, lung, liver, kidney, vertebrae, and veins. Limb buds are first apparent at E10.5, and individual hind- and forelimb digits become discernible at E15.5. The spine first appears as a faint echogenic line at E12.5. Ossification of the vertebral elements, the face and skull bones, and ribs can be identified at E13.5 and becomes progressively more evident, more echogenic, and more defined. The echogenic lungs grow increasingly distinct from the more hypoechoic liver beginning at approximately E12.5. The fetal stomach and urinary bladder are somewhat identifiable beginning at E13.5, when they appear as hypoechoic structures [6].

The survival and growth of the fetus are critically dependent on the placenta. Placental growth is often neglected despite this critical role, and abnormal placental size is now recognized as an early predictor of poor fetal growth and poor outcome in human pregnancies. The mouse has been widely used as an animal model to better understand physiological human implantation and placentation and anomalies in these processes, owing to its significant morphostructural and histological homologies to the same features in humans $[19,20]$. Anomalous intrauterine growth has been defined as a critical risk factor for perinatal and childhood morbidity and mortality and for diverse adult-onset diseases including diabetes, cancer, and hypertension. We have used HFUS for the diagnosis of pregnancy and the staging of placental and fetal development in CD1 mice. Statistical analysis was performed to assess changes in embryonic and placental morphology in relation to the embryonic day. These data will permit the accurate staging of pregnancy, which is necessary if therapy is to be performed at a precise time and if morphological abnormalities are to be identified at specific embryonic stages in the evaluation of animal models of congenital diseases.

\section{Materials and Methods}

Animal studies were performed according to a protocol approved by the Italian National Institutes of Health and the Animal Welfare Regulation Committee of the University Federico II of Naples, in adherence to the Guide for the Care and Use of Laboratory Animals published by the US National Institutes of Health (NIH Publication No. 85-23, revised 1996). Thirty CD1 adult female mice were analyzed by HFUS during pregnancy from E6.5 to E16.5 to evaluate different morphometric parameters. At each gestational day, at least 5 dams were monitored, for a total of $\sim 200$ embryos examined. To reduce the number of animals needed for experimental purposes, more than one measurement was made in each embryo, with caution taken to limit the duration of anesthesia (Table 1). Embryonic day E0.5 of pregnancy was estimated by the presence of a vaginal plug after overnight mating.

During the ultrasound examination, pregnant mice were lightly anesthetized using isoflurane $(4 \%$ induction dose, $2.0-1.5 \%$ maintenance dose) plus oxygen $(1 \mathrm{~L} / \mathrm{min})$. The mean anesthesia time increased with gestational age (range 20-45 min/dam) because the scanning time was increased to accommodate the greater number of measurements that could be recorded as the fetuses matured. Body temperature was monitored with a rectal probe and maintained in a physiological range using an infrared lamp. Hair was removed from the dam's abdomen by shaving, followed by the use of a chemical hair remover; then, ultrasound gel was applied to the skin to facilitate sound transmission and to reduce contact artifacts.

Embryos were imaged through the maternal abdominal wall with a Vevo 770 instrument (VisualSonics, Canada). The $40 \mathrm{MHz}$ high-resolution linear transducer was used (focal length $6 \mathrm{~mm}$, depth of penetration 5-15 mm; resolution, 30-40 $\mu \mathrm{m}$ axial and $70-90 \mu \mathrm{m}$ lateral). Two to four embryos were imaged in a single session to avoid prolonged anesthesia. Images of implantation sites, developing organs and quantitative variables for monitoring fetal growth were obtained in cross-sectional two-dimensional mode (Bmode). All measurements were made by the same operator to ensure consistency during the experiment and to avoid excessive consecutive observations, which might damage the dam or its conceptus.

\section{Statistical Analysis}

Because it is not possible to measure each variable for the entire duration of pregnancy, the parameters were divided into three groups as a function of the time at which they were measured.

Group 1, from E7.5 to E9.5: decidual thickness, gestational sac length, gestational sac thickness, implantation site length, implantation site thickness, and crown-rump length;

Group 2, from E9.5 to E1 1.5: gestational sac length, gestational sac thickness, placental length, placental thickness, crown-rump diameter, and biparietal diameter; and 
Table 1. Number of embryos for each measurements.

\begin{tabular}{lll}
\hline & & \\
\hline Type of measurements & Gestational Age (Day) & Number of measured embryos \\
\hline Decidual thickness & E6.5-9.5 & 95 \\
Gestational sac length & E7.5-11.5 & 112 \\
Gestational sac thickness & E7.5-11.5 & 112 \\
Implantation site length & E6.5-E9.5 & 95 \\
Implantation site thickness & E6.5-E9.5 & 95 \\
Placental length & E9.5-E16.5 & 128 \\
Placental thickness & E9.5-E16.5 & 128 \\
Crown-rump length & E7.5-E14.5 & 152 \\
Abdominal circumference & E11.5-E16.5 & 81 \\
Antero-posterior abdominal diameter & E11.5-E16.5 & 81 \\
Latero-lateral abdominal diameter & E11.5-E16.5 & 81 \\
Occipital-snout length & E12.5-E16.5 & 61 \\
Biparietal diameter & E9.5-E16.5 & 126 \\
Subretinal space thickness & E12.5-E16.5 & 68 \\
Antero-posterior lens diameter & E12.5-E16.5 & 68 \\
Latero-lateral lens diameter & E12.5-E16.5 & 68 \\
Stomach diameter & E12.5-E16.5 & 59 \\
\hline
\end{tabular}

doi:10.1371/journal.pone.0077205.t001

Group 3, from E12.5 to E16.5: placental length, placental thickness, abdominal circumference, antero- posterior abdominal diameter, latero-lateral abdominal, diameter, occipital-snout length, biparietal diameter, subretinal space thickness, anteroposterior lens diameter, latero-lateral lens diameter, and stomach diameter.

Univariate analysis between each measurement and embryonic day was performed using Spearman's rank correlation (Rs). Continuous linear regression was adopted for multivariate analysis of significant parameters. All statistical tests were two-sided, and a $\mathrm{p}$ value of 0.05 was considered statistically significant. Statistical analyses were performed using MedCalc 12 (Ostend, Belgium).

\section{Results}

High-frequency ultrasound (HFUS) is a valuable tool for monitoring placental and fetal growth. From E6.5 to E15.5, at least 5 dams were examined on each embryonic day. At E16.5, only 2 dams were examined, and few qualitative and quantitative evaluations were performed. Morphometric evaluations were conducted from E6.5 to E16.5. The values collected at several gestational ages (mean $\pm \mathrm{SD}$ ) are reported in Table 2 .

Placental development begins in the blastocyst at E3.5, when the trophectoderm layer is segregated from the inner cell mass. At E5.5, HFUS revealed the early implantation site, which is characterized by the apposition of the trophectoderm blastocysts with the uterine epithelium (Fig. 1 a). At E6.5, the embryo was visualized inside the decidua as an echogenic ectoplacental cone region and a small echolucent cavity (Fig. 1 b). At embryonic day 7.5 , the three cavities of the embryo, the amniotic, the coelomic cavity, and the ectoplacental cavity, were detected (Fig. 1 c). At E8.5, the embryo appeared to not yet have rotated into the position assumed throughout the rest of gestation, and early cardiovascular activity was visualized (Fig. $1 \mathrm{~d}$ ). On day 9.5, the amniotic membrane, amniotic and yolk sac cavities, brain, cerebral ventricles and heart were visible (Fig. $1 \mathrm{e}-\mathrm{h}$ ). At E10.5, the umbilical cord, eye lens, vitreous humor and retina were also clearly visible (Fig. 2 a, b). In the Fig. 2 c, an example of a dead embryo at E10.5 is showed. At E10.5, the open neural tube was used as the marker of brain development, while at E12.5, the brain was more clearly defined, with the mesencephalic vesicle (future aqueduct) and the third ventricle clearly visible. At embryonic day 13.5, crown-rump length could be measured, and the placenta, thorax, abdomen (Fig. $2 \mathrm{~d}-\mathrm{f}$ ), eye lens and vertebral column were visible (Fig. 2 g, h). At E14.5, the telencephalic vesicle and future lateral ventricles could be detected, and the occipital-snout length measurement could be made (Fig. 2 i). The choroid plexus was not routinely easily visible until E15.5, and no other individual structures could be discerned [21]. Later during embryonic development, many other structures could also be visualized, including the developing paw and forelimb, claw (Fig. $3 \mathrm{f}$ ), abdomen, lung, liver, kidney and blood vessels. Beginning at E14.5, the skull and ribs appear to be mineralized, and the interventricular septum is completed (Fig. $3 \mathrm{a}-\mathrm{c}$ ); the same is true for vertebral elements, the humerus and the femur beginning at E15.5 (Fig. 3 d, e). At embryonic day 16.5, the dorsal aorta and corresponding Doppler spectral trace are clearly visible (Fig. $3 \mathrm{~g}$, h).

Linear regression analyses (Table 3 ) were performed for the 3 groups. In the first group, only implantation site length, gestational sac length, gestational sac thickness and crown-rump length were significantly parameters, whereas decidual thickness and implantation site thickness remained outside the model. In the second group, gestational sac length, gestational sac thickness, placental length, crown-rump length and biparietal diameter were significant, while only placental thickness was not included in the model. In the third group, biparietal diameter, occipital-snout length, antero-posterior lens diameter, stomach diameter and anteroposterior abdominal diameter are meaningful (expressive), but placental length, placental thickness, abdominal circumference, subretinal space thickness, latero-lateral lens diameter and laterolateral abdominal diameter were excluded from the model. The 
Table 2. Morphometric evaluation.

\begin{tabular}{|c|c|c|}
\hline Type of measurement & Measurement at first day (mean \pm SD) & Measurement at last day (mean \pm SD) \\
\hline Decidual thickness & $1,01 \pm 0,11 \mathrm{~mm}(\mathrm{E} 6.5)$ & $0,94 \pm 0,22 \mathrm{~mm}(\mathrm{E} 9.5)$ \\
\hline Gestational sac length & $0,90 \pm 0,27 \mathrm{~mm}(\mathrm{E} 7.5)$ & $5,28 \pm 1,52 \mathrm{~mm}(\mathrm{E} 11.5)$ \\
\hline Gestational sac thickness & $0,62 \pm 0,36 \mathrm{~mm}(\mathrm{E} 7.5)$ & $3,22 \pm 0,70 \mathrm{~mm}(\mathrm{E} 11.5)$ \\
\hline Implantation site length & $3,66 \pm 0,24 \mathrm{~mm}(\mathrm{E} 6.5)$ & $4,87 \pm 0,73 \mathrm{~mm}(\mathrm{E} 9.5)$ \\
\hline Implantation site thickness & $2,46 \pm 0,31 \mathrm{~mm}(\mathrm{E} 6.5)$ & $3,90 \pm 0,64 \mathrm{~mm}(\mathrm{E} 9.5)$ \\
\hline Placental length & $2,85 \pm 0,20 \mathrm{~mm}(\mathrm{E} 9.5)$ & $6,65 \pm 0,79 \mathrm{~mm}(\mathrm{E} 16.5)$ \\
\hline Placental thickness & $2,16 \pm 0,32 \mathrm{~mm}(\mathrm{E} 9.5)$ & $4,36 \pm 0,12 \mathrm{~mm}(\mathrm{E} 16.5)$ \\
\hline Crown-rump length & $0,50 \pm 0,21 \mathrm{~mm}(\mathrm{E} 7.5)$ & $10,6 \pm 1,90 \mathrm{~mm}(\mathrm{E} 14.5)$ \\
\hline Abdominal circumference & $2,98 \pm 0,24 \mathrm{~mm}(\mathrm{E} 11.5)$ & $5,89 \pm 0,23 \mathrm{~mm}(\mathrm{E} 16.5)$ \\
\hline Antero-posterior abdominal diameter & $3,42 \pm 0,24 \mathrm{~mm}(\mathrm{E} 11.5)$ & $6,68 \pm 0,52 \mathrm{~mm}(\mathrm{E} 16.5)$ \\
\hline Latero-lateral abdominal diameter & $2,52 \pm 0,39 \mathrm{~mm}(\mathrm{E} 11.5)$ & $5,09 \pm 0,22 \mathrm{~mm}(\mathrm{E} 16.5)$ \\
\hline Occipital-snout length & $5,23 \pm 1,1 \mathrm{~mm}(\mathrm{E} 12.5)$ & $7,84 \pm 0,59 \mathrm{~mm}(\mathrm{E} 16.5)$ \\
\hline Biparietal diameter & $0,98 \pm 0,14 \mathrm{~mm}(\mathrm{E} 9.5)$ & $4,84 \pm 0,13 \mathrm{~mm}(\mathrm{E} 16.5)$ \\
\hline Subretinal space thickness & $0,14 \pm 0,04 \mathrm{~mm}(\mathrm{E} 12.5)$ & $0,21 \pm 0,01 \mathrm{~mm}(\mathrm{E} 16.5)$ \\
\hline Antero-posterior lens diameter & $0,23 \pm 0,07 \mathrm{~mm}(\mathrm{E} 12.5)$ & $0,46 \pm 0,05 \mathrm{~mm}(\mathrm{E} 16.5)$ \\
\hline Latero-lateral lens diameter & $0,26 \pm 0,08 \mathrm{~mm}(\mathrm{E} 12.5)$ & $0,44 \pm 0,07 \mathrm{~mm}(\mathrm{E} 16.5)$ \\
\hline Stomach diameter & $0,46 \pm 0,14 \mathrm{~mm}(\mathrm{E} 12.5)$ & $0,75 \pm 0,24 \mathrm{~mm}(\mathrm{E} 16.5)$ \\
\hline
\end{tabular}

Legend: The crown-rump length (CRL) was measured as the maximum distance from the cephalic pole to the caudal pole.

The abdominal circumference $(\mathrm{AC})$ was calculated by averaging the antero-posterior and latero-lateral abdominal diameters. Abdominal diameters were measured from a transverse section of the fetal abdomen at the level of the stomach and the umbilical vein.

Occipital-snout length represents the distance from the occipital prominence to the mouth.

Biparietal diameter is the distance between parietal bones and is easy to obtain by placing the electronic caliper on the hyperechogenic limit of the bones.

Measurements of placental growth included placental length and placental thickness measured at the level of umbilical vessel insertion.

doi:10.1371/journal.pone.0077205.t002
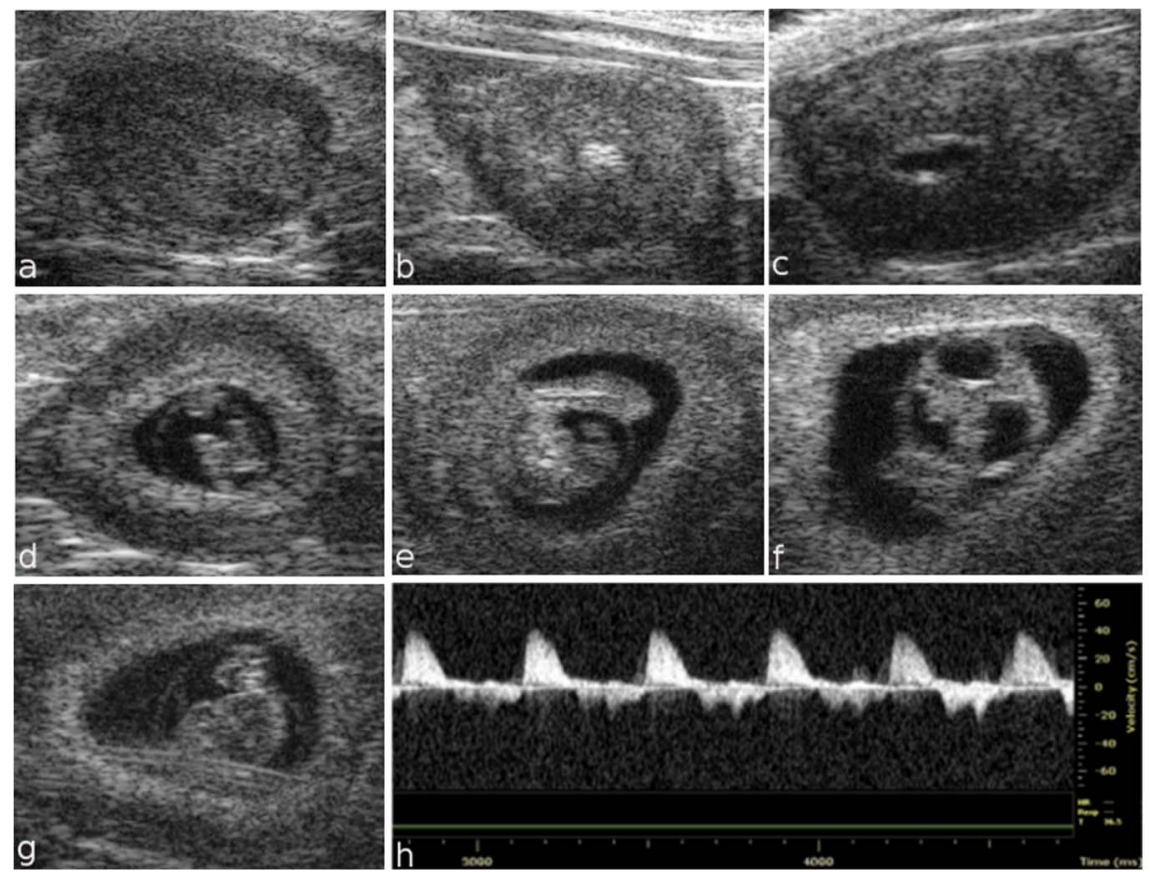

Figure 1. Ultrasound images of fetal development from embryonic day $\mathbf{5 . 5}$ to $\mathbf{9 . 5}$. Representative images of the implantation site at E5.5 showing the apposition of the blastocyst trophectoderm with the uterus (a). At E6.5, a small echolucent cavity containing the embryo is visualized inside the decidua (b). At E7.5, the amniotic and exocoelomic cavities and the ectoplacental cone region are discernable (c). At embryonic day 8.5, the heart, head and whole embryo are visualized (d). At E9.5, the amniotic membrane, yolk sac and cerebral ventricles are visible, while a Doppler spectral trace of ventricular inflow and outflow can be observed in the " $U$ shaped" heart tube $(e-h)$.

doi:10.1371/journal.pone.0077205.g001 

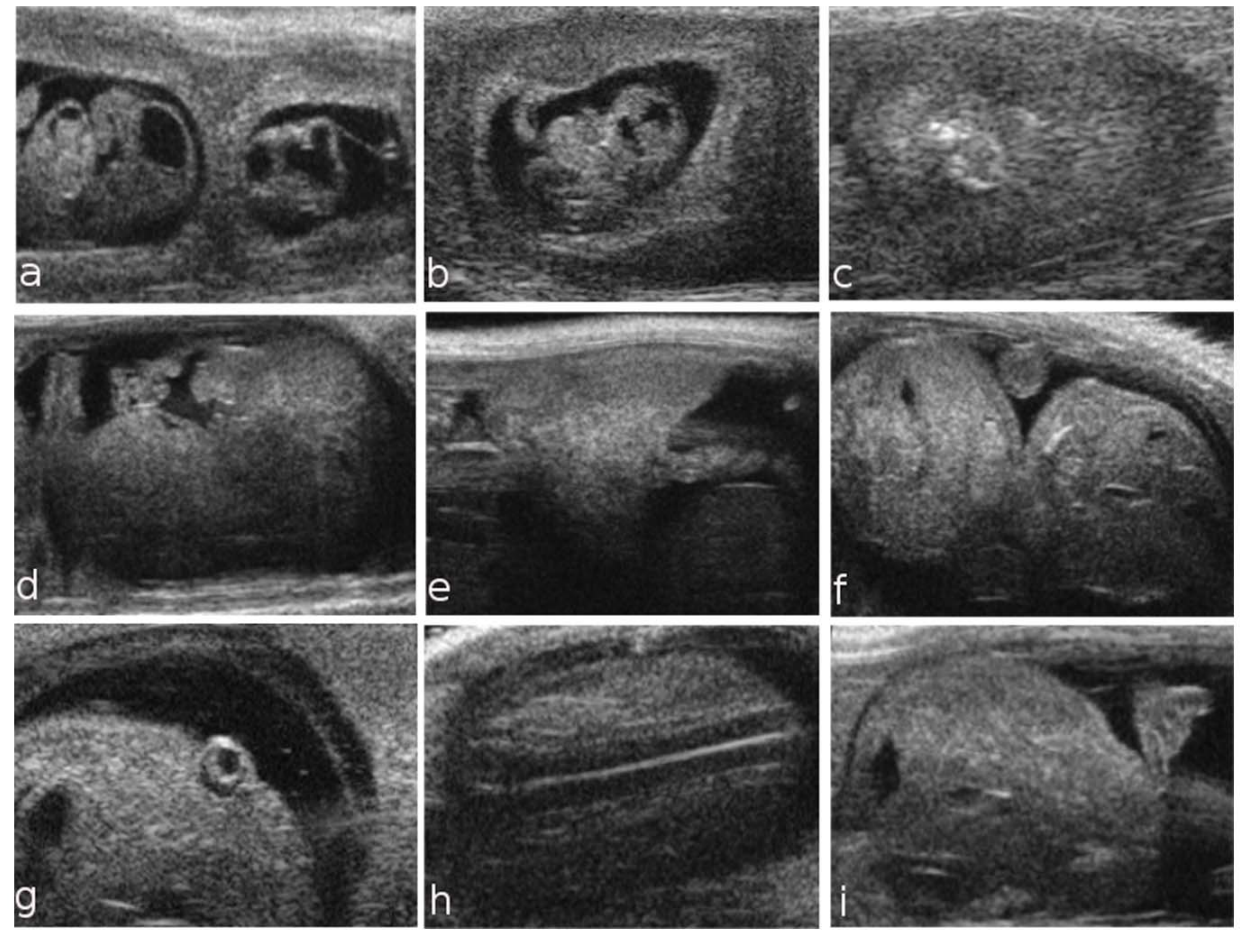

Figure 2. Ultrasound images of fetal development from embryonic day $\mathbf{1 0 . 5}$ to $\mathbf{1 4 . 5}$. At E10.5, it is possible to identify the umbilical cord, placenta and eyes ( $a, b)$; a sagittal view of a dead embryo is shown in (c). At embryonic day 13.5, crown-rump length (d), placental length and placental thickness (e) can be measured; the distinction between the fetal thorax and abdomen is defined ( $\mathrm{f}$ ); eyes with lens ( $\mathrm{g}$ ) and vertebral column (h) are visible. At E14.5, the occipital-snout length measurement can be reported (i). doi:10.1371/journal.pone.0077205.g002
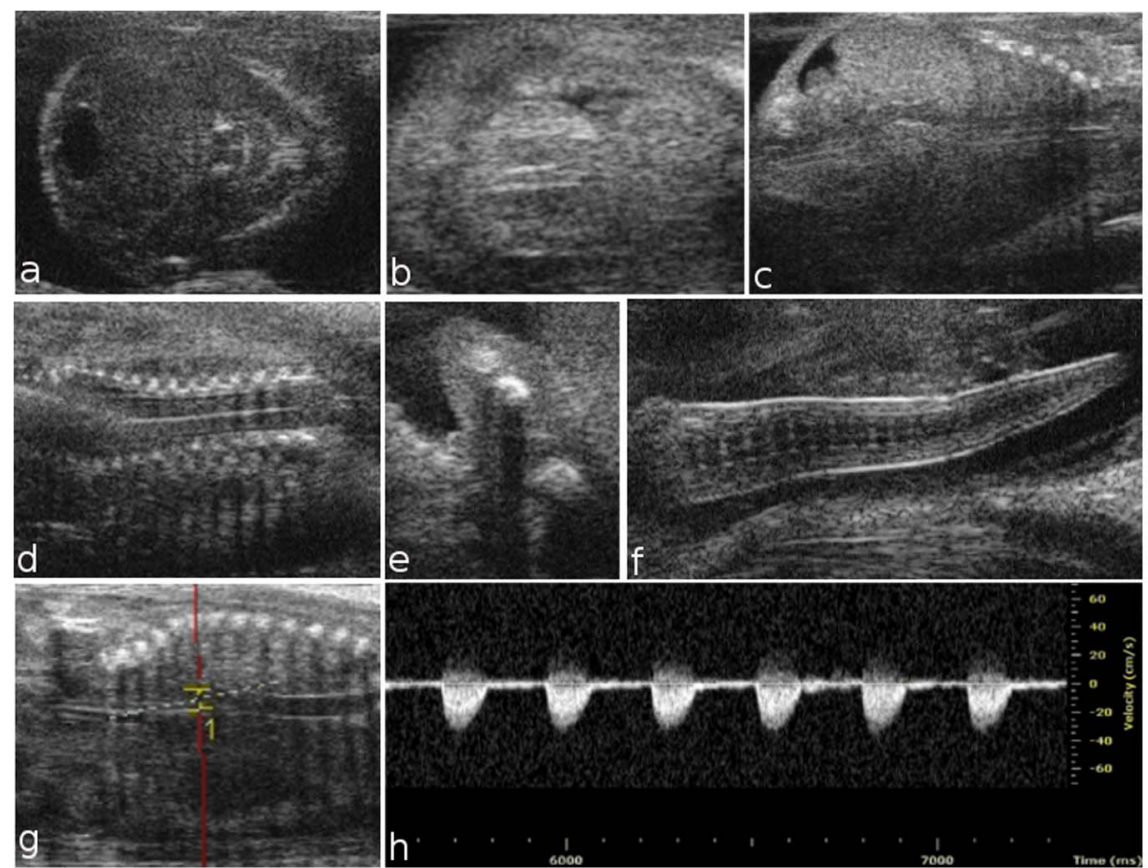

Figure 3. Ultrasound images of fetal development from embryonic day $\mathbf{1 4 . 5}$ to $\mathbf{1 6 . 5}$. At E14.5, progressive ossification is observed in the skull (a) and ribs (c), and the interventricular septum is completed (b). At 15.5, the development of the vertebral elements and humerus is complete $(d, e)$. At E16.5, the curl is clearly visible (f). Dorsal aorta and corresponding Doppler spectral trace at E16.5, which exhibits a rapid upstroke and return to zero velocity during diastole $(g, h)$.

doi:10.1371/journal.pone.0077205.g003 
Table 3. Spearman's correlation coefficient and significance of each embryonic parameter.

\begin{tabular}{|c|c|c|}
\hline Embryonic Structure & Correlation Coefficient & $p$ \\
\hline Decidual thickness & $-0,0827$ & 0,4257 \\
\hline Gestational Sac Length & 0,8983 & $<0,0001$ \\
\hline Gestational Sac Thickness & 0,8886 & $<0,0001$ \\
\hline Implantation Site length & 0,6234 & $<0,0001$ \\
\hline $\begin{array}{l}\text { Implantation Site } \\
\text { Thickness }\end{array}$ & 0,7102 & $<0,0001$ \\
\hline Placental Length & 0,8386 & $<0,0001$ \\
\hline Placental Thickness & 0,7364 & $<0,0001$ \\
\hline Crown-Rump Length & 0,9606 & $<0,0001$ \\
\hline $\begin{array}{l}\text { Abdominal } \\
\text { Circumference }\end{array}$ & 0,7426 & $<0,0001$ \\
\hline $\begin{array}{l}\text { Antero-Posterior } \\
\text { Abdominal Diameter }\end{array}$ & 0,8602 & $<0,0001$ \\
\hline $\begin{array}{l}\text { Latero-Lateral Abdominal } \\
\text { Diameter }\end{array}$ & 0,7395 & $<0,0001$ \\
\hline Occipital-snout length & 0,6523 & $<0,0001$ \\
\hline Biparietal Diameter & 0,9485 & $<0,0001$ \\
\hline $\begin{array}{l}\text { Subretinal Space } \\
\text { Thickness }\end{array}$ & 0,5559 & $<0,0001$ \\
\hline $\begin{array}{l}\text { Antero-Posterior Lens } \\
\text { Diameter }\end{array}$ & 0,5787 & $<0,0001$ \\
\hline $\begin{array}{l}\text { Latero-Lateral Lens } \\
\text { Diameter }\end{array}$ & 0,6255 & $<0,0001$ \\
\hline Stomach Diameter & 0,4617 & 0,0002 \\
\hline
\end{tabular}

doi:10.1371/journal.pone.0077205.t003

best-fit regression coefficients and standard error for the three groups of variables are shown in Table 4.

\section{Discussion}

Over the past several years, a number of authors have demonstrated the effectiveness of HFUS for staging fetal mouse gestational age and for the evaluation of morphological development in utero [22-25], particularly of the central nervous system, eye, and heart, in mouse embryos. HFUS appears to be a versatile tool for the detailed study of the morphogenesis of various organs. The disadvantages of HFUS are generally related to the advanced stages of gestation; some fetuses cannot be visualized or are not in an appropriate orientation or position for accurate measurements. After embryonic day 14.5, it might be difficult to obtain crownrump length measurement, but alternative indexes of fetal size, such as head diameter, can be used at this stage. In fact, occipitalsnout length and biparietal diameter of the fetal head were easily recorded in both longitudinal and transversal planes in a symmetric section of the skull. Beginning at E12.5, another anatomical structure available for measurement is the orbital diameter. This could be a useful indirect marker of brain development in mouse models because it has been reported that slow ocular growth is associated with delayed cerebral development in human fetuses [7]. Some authors showed that HFUS could affect brain development at certain frequencies [18,22], but we determined that ultrasound performed under isoflurane anesthesia during organogenesis has no significant effects on birth weight or postnatal growth; in fact, no deaths of the anesthetized pregnant female mice occurred in our study, and embryonic or fetal death was rare. The gestation length, mean birth weight and mean number of pups per litter were not affected by ultrasound or anesthesia exposure. Additionally, the ability to determine gestational age would also allow determination of the expected time of parturition, an advantage in breeding programs with mouse strains in which delivery is difficult for the dam. The exact time of fertile mating in mice is often unknown, so the ability to monitor and measure fetal organs would allow more accurate estimation of gestational age.

Table 4. Best-fit regression coefficients and standard error for the three groups of variables.

\begin{tabular}{|c|c|c|c|c|c|}
\hline Embryonic day & model & Set of parameters & Coefficient & Standard error & $p$ \\
\hline \multirow[t]{5}{*}{ E7.5-E9.5 } & Group 1 & Implantation site length & 0,1343 & 0,05011 & 0,0092 \\
\hline & & Gestational sac thickness & 0,1853 & 0,07057 & 0,0107 \\
\hline & & Gestational sac length & 0,4520 & 0,06622 & $<0,0001$ \\
\hline & & Crown-rump length (CRL) & 0,4988 & 0,1061 & $<0,0001$ \\
\hline & & Constant & 6,3475 & & \\
\hline \multirow[t]{6}{*}{ E9.5-E11.5 } & Group 2 & Gestational sac thickness & 0,1270 & 0,03938 & 0,0022 \\
\hline & & Gestational sac length & 0,07384 & 0,02222 & 0,0016 \\
\hline & & Placental length & 0,2023 & 0,05277 & 0,0003 \\
\hline & & Biparietal diameter & 0,2534 & 0,08666 & 0,0051 \\
\hline & & Crown-rump length (CRL) & 0,2066 & 0,02376 & $<0,0001$ \\
\hline & & Constant & 7,9073 & & \\
\hline \multirow[t]{6}{*}{ E12.5-E16.5 } & Group 3 & Biparietal diameter & 0,7009 & 0,1206 & \\
\hline & & Occipital snout length & 0,1444 & 0,06992 & \\
\hline & & Antero-posterior lens diameter & 1,5952 & 0,6204 & \\
\hline & & Stomach diameter & 1,2595 & 0,4298 & \\
\hline & & Antero-posterior abdominal diameter & 0,4805 & 0,08862 & \\
\hline & & Constant & 6,7749 & & \\
\hline
\end{tabular}


We have shown that fetal and placental growth can be documented during pregnancy in mice using HFUS. We used the outbred mouse strain CD1 because it is the most widely used in basic reproductive biology studies due to the genetic variability in the experimental population. Our results can provide useful data for establishing growth curves for these structures in the strain examined, which will be a valuable tool for research in mouse pregnancy. In particular, we suggest three groups of parameters that can be used to predict the embryonic day among those parameters that can be measured at a given gestational age. HFUS is a valid methodology for the surveillance and measurement of mice embryonic development from very early stages of pregnancy. HFUS is performed in real time and is both easy and rapid. This technique is favorable for animal well being, allowing serial

\section{References}

1. Johnsen SL, Wilsgaard T, Rasmussen S, Sollien R, Kiserud T (2006) Longitudinal reference charts for growth of the fetal head, abdomen and femur. Eur J Obstet Gynecol Reprod Biol 127: 172-185.

2. Mullis PE, Tonella P (2008) Regulation of fetal growth: consequences and impact of being born small. Best Pract Res Clin Endocrinol Metab 22: 173-190.

3. Dickinson ME (2006) Multimodal Imaging of Mouse Development: Tools for the Postgenomic Era. Dev Dyn 235: 2386-2400.

4. Spurney CF, Leatherbury L, Lo CW (2004) High frequency Ultrasound Database Profiling growth development and cardiovascular function in C57Bl/ 6J mouse fetuses. J Am Soc Echocardiogr 17: 893-900.

5. Phoon CKL (2006) Imaging Tools for the Developmental Biologist: Ultrasound Biomicroscopy of Mouse Embryonic Development. Pediatr Res 60: 14-21.

6. Brown SD, Zurakowski D, Rodriguez DP, Dunning PS, Hurley RJ, et al. (2006) Ultrasound diagnosis of mouse pregnancy and gestational staging. Comp Med 56: $262-271$.

7. Mu J, Slevin JC, Qu D, McCormick S, Adamson SL (2008) In vivo quantification of embryonic and placental growth during gestation in mice using micro-ultrasound. Reprod Biol Endocrinol 6: 34-47.

8. Pallares P, Gonzalez-Bulnes A (2008) Non-invasive ultrasonographic characterization of phenotypic changes during embryo development in non-anesthetized mice of different genotypes. Theriogenology 70: 44-52.

9. Dickinson H, Griffiths T, Walker DW, Jenkin G (2008) Application of clinical indices of fetal growth and wellbeing to a novel laboratory species, the spiny mouse. Reprod Biol 8: 229-243.

10. Zhao H, Wong RJ, Doyle TG, Nayak N, Vreman HJ, et al. (2008) Regulation of maternal and fetal hemodynamic function by heme oxygenase in mice. Biol Reprod 78: 744-51.

11. Turnbull DH (1999) In utero ultrasound backscatter microscopy of early-stage mouse embryos. Comput Med Imaging Graph 23: 25-31.

12. Russo M, Meomartino L, Scarfo M, Volpe P, Cocchia N, et al. (2007) Pregnancy detection in mice using ultrasound. Vet Record 160: 446-447. ultrasound examinations to be obtained on subsequent days. Moreover, the short duration of ultrasound examination avoids the risk of inducing changes in pre- and post-natal growth and development [8]. In conclusion, HFUS is an important phenotyping tool for embryonic mouse research and can be used to answer important questions in developmental biology.

\section{Author Contributions}

Conceived and designed the experiments: AG MS AB. Performed the experiments: MR ARDC SG MG. Analyzed the data: RL AR. Contributed reagents/materials/analysis tools: SA. Wrote the paper: AG ARDC. Helped in translating the manuscript: SP. Supervise the use of Vevo 770 High Frequency Ultrasound: MM.

13. Slevin JC, Byers L, Gertsenstein M, Qu D, Mu J, et al. (2006) High resolution ultrasound-guided microinjection for interventional studies of early embryonic and placental developmental in vivo in mice. BMC Dev Biol 6: 10.

14. Foster FS, Pavlin CJ, Harasiewicz KA, Christopher DA, Turnbull DH (2000) Advances in ultrasound biomicroscopy. Ultrasound Med Biol 26: 1-27.

15. Foster FS, Zhang M, Duckett AS, Cucevic V, Pavlin CJ (2003) In vivo imaging of embryonic development in the mouse eye by ultrasound biomicroscopy. Invest Ophthalmol Vis Sci 44: 2361-2366.

16. Foster FS, Zhang MY, Zhou YQ Liu G, MehiJ, et al. (2002) A new ultrasound instrument for in vivo microimaging of mice. Ultrasound Med Biol 28: 11651172 .

17. Srinivasan S, Baldwin HS, Aristizabal O, Kwee L, Labow M, et al. (1998) Noninvasive, in utero imaging of mouse embryonic heart development with 40MHz echocardiography. Circulation 98: 912-918.

18. Kulandavelu S, Qu D, Sunn N, Mu J, Rennie MY, et al. (2006) Embryonic and Neonatal Phenotyping of Genetically Engineered Mice. ILAR J 47: 103-117.

19. Carson DD, Bagchi I, Dey SK, Enders AC, Fazleabas AT, et al. (2000) Embrio Implantation. Dev Biol 223: 217-237.

20. Watson ED, Cross JC (2005) Development of structures and transport functions in the mouse placenta. Physiology 20: 180-193.

21. Brown SD, Zurakowski D, Rodriguez DP, Dunning PS, Hurley RJ, et al. (2006) Ultrasound diagnosis of mouse pregnancy and gestational staging. Comp Med 56: 262-271.

22. Mazze RI, Wilson AI, Rice SA, Baden JM (1985) Fetal development in mice exposed to isoflurane. Teratology 32: 339-345.

23. Lim HJ, Wang H (2010) Uterine disorders and pregnancy complications: insights from mouse models. J Clin Invest 120: 1004-1015.

24. Carter AM (2006) Animal Models of Human Placentation. Placenta 21: 41-47.

25. Georgiades P, Ferguson-Smith AC, Burton GJ (2002) Comparative Developmental Anatomy of the Murine and Human Definitive Placentae. Placenta 23: 3-19. 